\title{
Information models of structures and modeling in construction
}

\author{
Vladimir Sokolnikov ${ }^{1 *}$ [0000-0003-3768-2079], Irina Osipenkova ${ }^{1}$ [0000-0003-4568-0030], Olga \\ Stupakova ${ }^{10000-0002-2595-6100]}$, Roman Motylev ${ }^{10000-0002-2026-0390]}$, and Roza Nurgalina ${ }^{10000-0003-}$ \\ 4467-259X]
}

${ }^{1}$ Saint Petersburg State University of Architecture and Civil Engineering, 190005, 2-ya

Krasnoarmeiskaya st., St. Petersburg, Russia

\begin{abstract}
The study of the basics of modeling of technical processes and objects forms the scientific basis for substantiating theoretical conclusions and postulates, classifying and developing the structures of theoretical models and justifying practical methods of preparation and implementation of (construction) production, which, in turn, influence the adoption of legal and regulatory innovations and statements. The article analyzes the types of modeling and models, scientific, technical, legal and regulatory contexts of the use of the concepts of «information modeling» in construction, and compares them with classical methods and tasks of modeling technical systems in solving scientific and engineering problems. The classification of scientific methods of modeling and models of technical objects and systems in construction is carried out. Examples of analytical models from the point of view of their classification and purpose are considered. It is shown that in the absence of clear scientific definitions of the concepts of «information», «model» and «modeling», given in terms of the actual field of knowledge, or application, the concept of «information model» comes into conflict with scientific and engineering ideas about the properties and purpose of the model. An approach is proposed to avoid the misuse of the concepts of «model» and « modeling».
\end{abstract}

Keywords: information modeling, information support of construction, classification of types of modeling and models of technical systems.

\section{Introduction}

Currently, various aspects of the problem of modeling construction processes, as well as modeling in construction design, are studied by many Russian and foreign researchers. The very concepts of «model» and «modeling» are used in such fields as the educational process of educational institutions, legal and administrative practice, and technical regulation. Currently, a number of standards and regulations in the field of information modeling have been prepared at the level of the Government and the Federal Agency for Technical Regulation and Metrology of the Russian Federation (GOST R 10.01.002 «Information modeling in construction» 2017, etc.). At the same time, the situation with the

\footnotetext{
* Corresponding author: vschief@yandex.ru
} 
definition of the ownership, essence and purpose of information modeling is quite contradictory. Available definition of the building information model ${ }^{1}-$ «a set of interrelated information ... about the object of capital construction...». It cannot be considered either as scientific or as engineering, since any actual phenomenon or process in any field of human activity can be recorded (determined) as «a collection of interrelated information and, ultimately, as a collection of material information carriers». The following definition of a model does not clarify the situation: «A model is a set of data created according to the structure underlying the data schema that meets certain requirements $»^{2}$. It seems obvious that the word «totality» or «set» in each subject area of knowledge is revealed through narrow special terms that specify its meaning in scientific or industrial characteristics. The situation is further complicated by the fact that $\langle B I M »$ and $« 4$ or more d»types of modeling are used as synonyms for «information» modeling, and also without their proper definitions. BIM, in form, means an abbreviation of the sonorous slogan «Building. Information. Model (modeling)». In its meaning lays the motto of the development of the construction industry based on the development and improvement of current models and their information (software) support. «4 or more» $\mathrm{d}$ «modeling» is difficult to interpret from a scientific and engineering perspective. But will explain it further. It should also be noted that the very definition of information is still the subject of scientific controversy. Thus, the content of this article is the justification of the answer to the questions: «what is a model?», «what is modeling?», «what is information?» and how to understand them from a scientific and engineering points of view and apply the concepts of «information modeling» (hereinafter-IM) and «information model» (hereinafter-IMS).

\section{Materials and methods}

In the database of the scientific and technical library eLIBRARY.ru at the time of this writing, there are 47941 works dedicated to the modeling problem. About 28000 of them were devoted to mathematical modeling, about 9,000 were devoted to simulation, and 217 publications were devoted to «information modeling of structures», made in the period from 2011, that is, less than $0.5 \%$ of the total volume of publications.At the same time, there is a rapid development of information technologies for construction design and mobile means of communication and data processing. Based on the analysis of the publications presented in eLIBRARY.ru, we can distinguish the following areas of research in construction, using modeling and, in particular, « information modeling. Various types of modeling are studied: technological processes [1-2], tasks of organization and management of construction production [3-6], design and construction systems [7], methods of implementation of innovative projects [8], planning of risks of failure of construction deadlines, optimization of calendar plans [9-10], sequence of development of reconstruction objects [11-12], optimization of design and construction of buildings by time parameter [13-14], reproduction of urban infrastructure [15], and others.

Research with the use of «information» modeling is conducted in the following areas:

- imitation of calendar planning in building information modeling programs [16]. The article proposes a methodology for the economic assessment of the entire building at any time of its design based on the use of the Revit platform;

- reviews, analysis and study of $4 \mathrm{~d}$ tools, BIM information modeling; innovative proposals and integration of BIM technologies; a significant number of publications are devoted to these issues;

${ }^{1}$ «Urban Planning Code of the Russian Federation» dated 29.12.2004 No. 190-FZ (as amended on 23.04.2018)

${ }^{2}$ GOST R 10.02 2019.p. 3.1. 20 
- construction design, development of project and working documentation in the REVIT program - the overwhelming number of publications;

- interaction of participants in the construction process through the EMC[17-18];

- development of the construction organization by IM [19-21]. of IM.

Fig. 1 shows the percentages of publications recorded at the time of writing in the areas

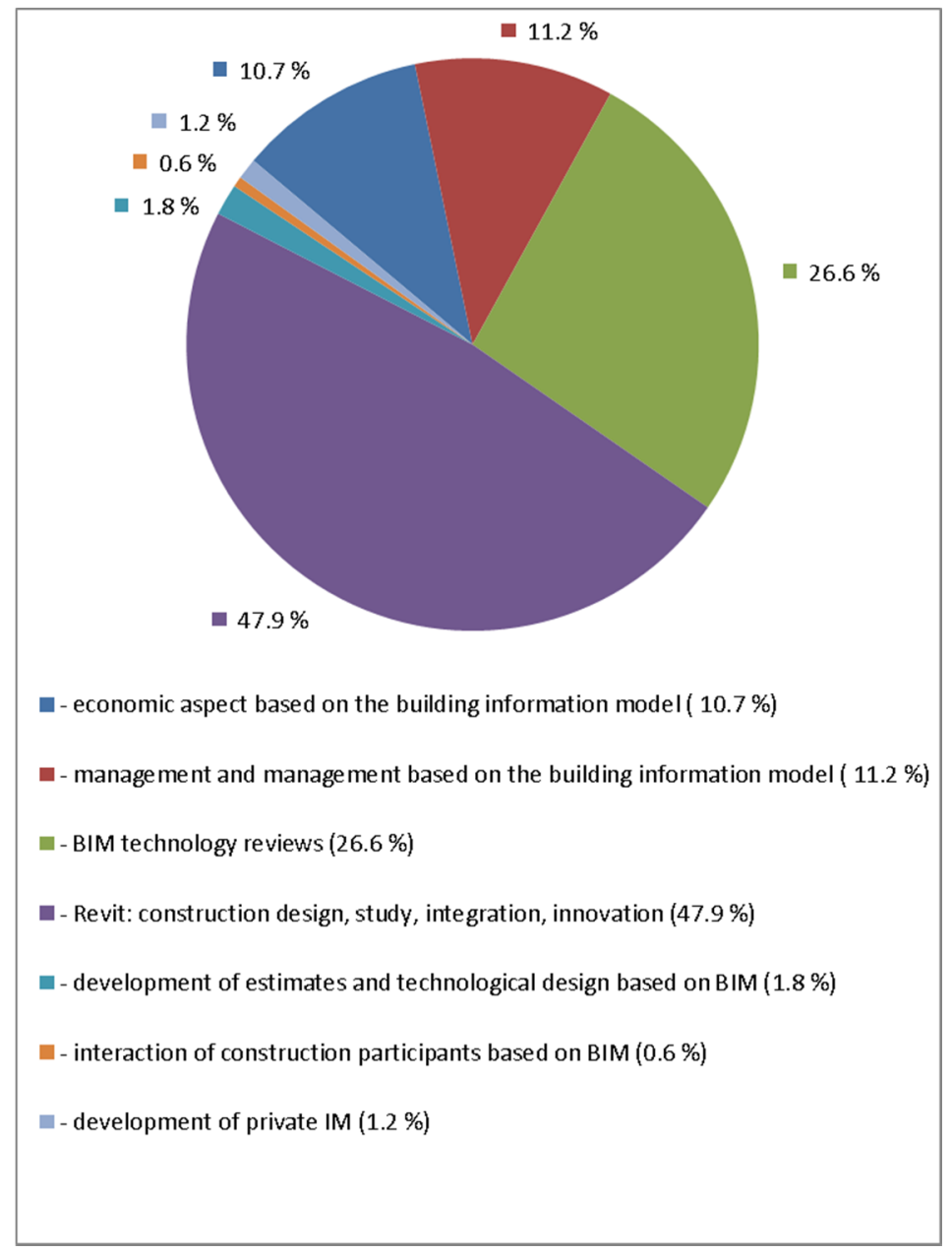

Fig. 1. Structure of information modeling research.

The analysis of Fig. 1 shows that attempts to develop, scientific, or engineering understanding and description of the IMS are not made, and under the terminology of modeling, the tasks of variant construction design and the release of working documentation are mainly solved. In the vast majority of publications, IT is understood as research on the capabilities of software for building design tasks. In the «Revit: Design, Integration, Innovation» chart sector, the design and research of the Revit package account for approximately $45 \%$ of publications. Review publications on the topic of IM, as a rule, contain a description of the problems of «information» modeling and its implementation. At the same time, it should be noted that in these publications and other sources available to the authors, scientific or engineering definitions of «information model of construction», as well as the concept of «information modeling», are not found, and the very use of the 
concepts of «model» and «modeling» is often incorrect in relation to the described tasks, problems and content of publications. So, for example, variant design in publications is called «modeling», or «BIM modeling», or« $4 \mathrm{~d}$ modeling». That is, the design process has now ceased to be terminologically described as an engineering task, but it has also not become a scientific task, since it is not in fact one. Such a situation with scientific and technical publications devoted to THEM shows that the use of the concepts of «information» model, or «modeling» in engineering and scientific practice of construction currently does not have a proper scientific or engineering justification and practical application, but, at the same time, is actively present, mainly in regulatory and administrative documentation ${ }^{3}$.

The implementation of the scientific and engineering definitions of the concepts of «model», «modeling» and «information» before and now meets with serious difficulties. For example, Academician Nemchinov gives the following general definition of the model. «The model is a means of identifying any objectively acting system of regular connections and relations that take place in the studied real reality» [22]. Other definitions of the model are also known. From the above definition, it follows that the «model» is a tool for studying the objective relations and relations (regularities) of the observed deterministic object. From the point of view of this definition, permits, working documentation for a structure, executive documentation, as well as construction in progress, or a structure in operation are different objects of different nature, connections and relationships, and, therefore, it is impossible to build a model of these objects. From a practical point of view, the concept of a model is widely used in the sense of «a primary sample of the result of the application of technologies that meets the requirements». This is a narrow engineering and production area of interpretation of the concept: (for example, a model for casting a part, or a photo model). Using the concept of «model» in this sense gives an early accurate idea of the future properties of the object being modeled. In construction, this interpretation of the concept of «model» is revealed through a special engineering term «a set of working (design) documentation for the construction of a structure $\rangle^{4}$.

The «information model», as defined in the urban planning code, is essentially a replenished data set, probably structured for some range of tasks of their automated processing. Previously, such an array was called «databases with different architectures», which were suitable for automatic sorting and filtering of data. Later, equipped with sets of processing algorithms and display modules, they became known as «knowledge bases». The initiative to create and use them came from the developers themselves. Later, this trend was developed by the administrative initiative: such concepts as «process» and «objectoriented» approach, «innovation», «digitalization», «information», «BIM» and «4d» modeling, which are vague from scientific and engineering points of view, have appeared not as a result of corresponding scientific or engineering achievements, or the discovery of new classes of engineering or scientific tasks, but in the form of slogans that use and often distort scientific and engineering terminology. Looking ahead, we can say that everything that is associated with IT relates to the information support of methods for solving various problems, including various types of modeling.

As mentioned above, in the scientific context, the model «should explain», and in the engineering and production context -«serve as a model». It is clear that the engineering interpretation of the concept of a model is not suitable for «information» modeling, so we turn to the scientific understanding of the model and modeling. According to the theory

\footnotetext{
${ }^{3}$ Decree of the Government of the Russian Federation of 15.09 .20 «On the maintenance of the information model»., GOST R 10.01.0001 (2) 2019, GOST R 10.02.0001(5) 2019, Order of the Ministry of Transport of the Russian Federation No. AK-177r of 17.09.2020, and so on.

${ }^{4}$ Resolution of the Government of the Russian Federation of 16.02.2008 N 87 «On the composition of sections of project documentation..».
} 
[23], «...the modeling is based on the similarity theory, which states that absolute similarity can only take place when one object is replaced by another exactly the same. The similarity theory excludes a complete transformation of the nature of the object during the observation period. Therefore, as one of the first signs of classification of types of modeling (and hence models) you can choose the degree of completeness of the model and divide the models according to this feature into complete, incomplete, and approximate ones». Complete models include all the parameters that affect the characteristics under study and describe all the dependencies associated with them. Incomplete models are set explicitly and explain the dependencies of some object characteristics on changes in the selected parameters. Approximate models explicitly establish the main relations of the objective function and the general nature of the dependencies in a certain range of argument values. Depending on the nature of the objects and processes under study, all types of modeling can be divided into deterministic and stochastic, static and dynamic, discrete and continuous. Deterministic modeling displays processes and phenomena in which the absence of any random effects is assumed; stochastic modeling displays probabilistic processes and events. In this case, a number of realizations of a random process are analyzed and the average characteristics, i.e., a set of homogeneous realizations, are estimated. Static modeling is used to describe the behavior of an object at a given time, and dynamic modeling reflects the behavior of an object over time. Discrete modeling is used to describe processes that are assumed to be discrete, respectively, continuous modeling allows you to reflect continuous processes in systems, and discrete-continuous modeling is used for cases where you want to highlight the presence of both discrete and continuous processes. It is important to note that the concept of «continuity» is used not only in the sense of»in time», but also in the sense of the fact of recorded changes in any real parameter. For example: the process of receiving materials to the warehouse is continuous in the parameters «invoice processing», «unloading» and discrete in the time parameter.

Mathematical modeling. Mathematical modeling is understood as the process of establishing the correspondence of certain mathematical objects to a given real object: formulas, systems of equations, tables of argument values and functions themselves, an explicit and implicit way to set a function, boundary conditions, etc., called a mathematical model, and the study of this model, which allows us to obtain the characteristics of the real object under consideration. The type of mathematical model depends both on the nature of the real object, and on the tasks of studying the object and the required reliability and accuracy of solving this problem. The following types of mathematical models are distinguished:

- «continuously-deterministic» models (D-schemes). They are used for the analysis of systems functioning continuously with respect to a certain parameter, for example: automatic control systems (according to the time parameter). The models are presented in the form of integro-differential equations;

- discrete-deterministic (F-schemes) for the analysis of systems by methods of the theory of automata, which treats the system as an object (finite automaton (finite)), which has a finite number of internal states and input signals, and to which input signals are supplied and from which output signals are removed (in mathematics, they correspond to the apparatus of one-dimensional matrices-the state vector);

- discrete-stochastic (P-schemes) for the analysis of systems that exhibit statistically regular random behavior. In this case, the system is defined as a probabilistic (stochastic English probabilistic) automaton, i.e., as a clock converter with memory, the functioning of which in each clock cycle depends only on the state of memory in it (without taking into account the initial and boundary (boundary) conditions) and can be described by a statistical regularity (2x and more dimensional matrices); 
- continuous-stochastic (Q -schemes from English «Queueing»)for queuing systems, for example: supply flows, flows of parts on the conveyor, requests for processing information on a computer from remote terminals (Internet). The random appearance of requests and the completion of service at a random timearetypical for the operation of such systems, i.e. the stochastic nature of their functioning (methods of probability theory).

Based on the analysis performed, the following definitions of the concepts «information», «modeling» and «model» can be given, which should be used in the description and formation of subject scientific and engineering models.

Information represents signals of various nature about the state of the environment and the object itself, formed according to the algorithms of functioning, as well as stored in the object, which are transformed by it into mechanisms for regulating the order of functioning. When functioning, objects consume, process, and produce various forms of information. Information is an attribute of the purposeful functioning of both the object itself and its model.

Modeling (scientific) is setting the boundaries of changes in the properties of an object with changes in the specified ranges of values of the actual parameters of the object, or the environment, performed using the object model. The determination of the boundaries and the nature of the changes, as well as the study of the obtained dependences on the extremes, will give the form of the nonlinearity of the dependence in the form of a mathematical function. Modeling (engineering) is a computational and graphical justification, or a visual representation of the future appearance, or properties of an object. Sometimes the word «modeling» is replaced directly by the names of the methods and the object of modeling. For example: «graphical method...».

Model is the material elements, the order, conditions and limitations of their functioning, determined by the nature of the object being modeled and the problem being solved, expressed in the corresponding interrelated logical, graphic and mathematical symbols and put in accordance with the real object or phenomenon, if the study of any property of a real object, or a group of dependent properties of one nature is excessively expensive, or practically impracticable. This definition of the model contains a format for recording special terms that form the meaning of the model and allows you to formulate its name and place in the subject area of research. The purpose of such a model is to explain the dependence of changes in the properties of an object when the values of actual parameters change, or the order of functioning of the elements that model the object change. Classification of models according to their scientific or engineering specialization allows you to establish modeling methods. For example: «geo-mechanical model of load distribution from foundation to foundation». Or: «heat engineering model of enclosing structures to resist heat transfer». The purpose and methods of the «aerodynamic model of the resistance of load-bearing and enclosing structures to wind loads»are also revealed. The computational and graphical model of the organization of construction in engineering practice is called the «calendar schedule of work» and «construction master plan».

The classification of modeling types given in [23] is revised by the authors of the article on the basis of their definitions of «information», «modeling» and «model» and includes a hierarchy of modeling types for complete, incomplete and approximate models, shown in Fig. 2.

The classification of modeling types shown in Fig. 2, as well as the analysis of their properties, show that the types of modeling are classified depending on the nature of the object being modeled, the tasks and methods of studying its properties, which is reflected in the classification features of modeling. The types of modeling that are supposed to be used in the study of the object complement each other, taking into account their hierarchy in the structure of Fig.2 and on their basis form the full subject name of the model. It should be 
noted that the model is always developed under the known conditions of the problem, and not vice versa.

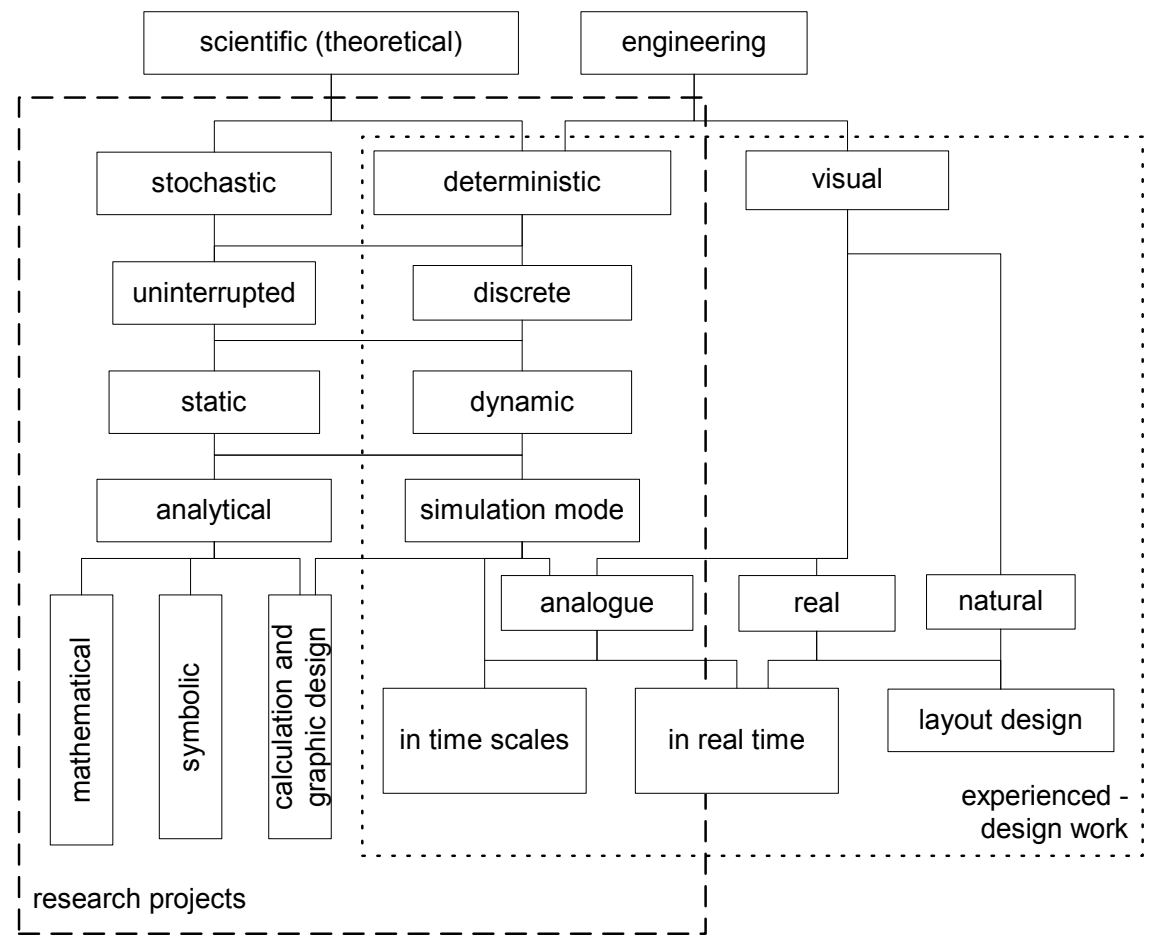

Fig. 2. Classification of modeling types.

As an example for comparing the types of modeling, let's take the continuity mathematical physics equation [24] and apply it to «4d» modeling in construction.

$\frac{d f(x, y, z)}{d t}=\frac{\partial f(x, y, z)}{\partial t}+A_{x} \frac{\partial f(x, y, z)}{\partial x}+A_{y} \frac{\partial f(x, y, z)}{\partial y}+A_{z} \frac{\partial f(x, y, z)}{\partial z}=\frac{\partial f(x . y . z)}{\partial t}+A \nabla f$

The equation (1) of the total time differential of the function $f(x, y, z, t)$ shows that the value of the function continuously changes with time and from point to point in space, which corresponds to the phenomena of transport: thermal conductivity, hydrodynamics, mechanics, electrodynamics. That is, (1) is a mathematical model of the continuity of the transport phenomenon and is intended for the study of transport phenomena and processes that are continuous in time and in a finite volume. Formally, the mathematical model (1) corresponds to the concept of « $4 \mathrm{~d}$ modeling», but the name of this model initially contains the main physical property, which is described by the model. The example of modeling in the field of construction follows directly from (1) [5]. The system of equations (2) is a continuously deterministic approximate model of the construction flow. The system of equations (2) in vector form describes the dependence of the increase in the construction readiness of the structure on the technological flow. The increment of construction readiness corresponds to the mass of structures assembled over a period of time. The increment in construction readiness is the result of the movement of structures under the action of $\mathrm{F}$ forces. The forces $\mathrm{F}$ act (applied to the structures) for a certain period of time:

$$
\left\{\begin{array}{l}
\operatorname{div} \mathrm{V}=4 \pi \rho_{\mathrm{k}} \\
\operatorname{rot} \mathrm{V}-\frac{\partial \rho_{k}}{\partial t}=4 \pi \mathrm{F}
\end{array}\right.
$$




$$
\operatorname{rot}_{z} \mathbf{V}=\frac{\partial V_{y}}{\partial x}-\frac{\partial V_{x}}{\partial y} ; \mathbf{F}=\operatorname{rot} \mathbf{A} ;
$$

where $\mathrm{V}$ is the velocity vector of the construction flow (construction of the building); $\operatorname{divV}$ - increment of construction readiness $\rho_{\mathrm{k}}(\mathrm{x}, \mathrm{y}, \mathrm{z})$ is the structural density of the structure on the private front (capture) - a scalar value, A-vector potential of the technological flow; $\mathrm{F}$ is the force vector of the technological flow of the mass transfer of structures, the vertical component of the rotor of which $\operatorname{rot}_{\mathrm{Z}} \mathrm{A}$ determines the vertical component of the speed $\mathrm{V}$ of the construction of the structure (construction flow), and therefore allows you to determine the time (duration) of the construction of the building to the upper design mark. That is, (2) and (3) are a model that explains the dependence of the increment of construction readiness on the intensity of the technological flow, on which the corresponding types of modeling of various characteristics of the structure, including the duration of construction work, are performed.

The examples of mathematical parts of deterministic models clearly show the meaninglessness of using adjectives that are disconnected from the essence of the problem and modeling methods to define the model.

\section{Results and discussion}

An extremely small number of noteworthy scientific and technical publications on the topic of «information» modeling of structures, in comparison with other types of modeling, indicates the isolation from scientific and engineering practice of attempts to apply the concept of «information modeling», replacing the concept of «information support» of scientific and engineering modeling of physical phenomena and processes in construction, construction design, as well as the organization of the construction of structures and control over it.

In the scientific and engineering literature that deserves attention, the authors have not found any definitions and facts of the description and use of «information»models of structures and«information» modeling. At the same time, sources have been found that classify a significant number of types of modeling, each of which requires information support, based on the analysis of the process of scientific knowledge, areas of scientific knowledge and engineering practice. According to these sources, any model without a mathematical part (model) is incomplete, or approximate (scientific), or industrial (engineering), and the name and description of the model is formed based on the studied properties of the object and the corresponding modeling methods, among which «information» is absent and is not applicable due to the non-relevance of the concept of «information». Attributing a model only as «informational», as well as «material», or «temporal»does not make scientific and engineering sense, since any model always has «informational», «material», and «temporal» attributes.

The production «information» model of a building can be conditionally accepted as a set of working documentation of a construction object, but such a «model» is not classified according to existing scientific features, and the replacement and duplication of production concepts destroys the established contexts of their use. Other types of construction documentation and data do not form models, but are only forms of structured data sets that require further processing with the use of information technologies.

Based on the above, it can be argued that the introduction and constructive use of clarifying definitions to the concept of «model» should be only after checking their compliance with the classification characteristics of the types of modeling performed with the help of these models. 


\section{Conclusion}

1. The official use in engineering, scientific, educational and administrative activities of the concepts of «information», «model», «modeling», as well as other engineering and scientific concepts without their definition in terms that reflect the level of development of the subject areas of science and technology leads to confusion and confusion of concepts, which destroys the dialectical and scientific approach to research and technology development. «Informational» is not a scientific or engineering classification feature of the model and the type of modeling.

2. Based on the above, the authors considered it appropriate to develop and adopt GOST, or other document of the scientific and engineering community, establishing a classification based on the model of Fig.2 types of modeling and their corresponding scientific and engineering models, containing strict scientific and technical system definitions of these concepts, made in terms that reflect, as it is often necessary, the intersectoral level of development of science and technology.

3. When developing the above-mentioned document, it is necessary to be guided, first of all, by the scientific and technical ideas about models and modeling set out in the article, which should be the basis for the terminology of both classification and their definitions and interpretations. In this case, the emergence and promotion of pseudo-scientific, pseudotechnical and pseudo-educational disciplines, as well as empty administrative initiatives that destroy scientific and engineering approaches to decision-making, will be excluded. This document will also protect a wider range of scientific and engineering concepts from misuse in unacceptable contexts.

\section{References}

1. O. A. Pobegailov, A. V. Shemchuk,Modeling of technological processes in the organization of construction productio, Online Journal of Science, 4, 4 (2012) URL: https://cyberleninka.ru/article/n/modelirovanie-tehnologicheskih-protsessov-priorganizatsii-stroitelnogo-proizvodstva (last accessed: 25.04.2021 г.)

2. I. L.Abramov, Modeling of technological processes in low-rise construction. Abstract. (Moscow, 23, 2007)

3. V. V. Sokolnikov,Decomposition of the problem of ensuring organizational and technological reliability of construction on the basis of classification of definitions of the conceptual field «organization of construction», Bulletin of Civil Engineers, 3(74), 87-93 (2019)

4. V. V. Sokolnikov, Improvement of operational planning of construction and installation works and their resource support on the basis of a unified information management environment(Autoref. dis. ... Candidate of Technical Sciences, St. Petersburg, 2017)

5. V. V. Sokolnikov, Modeling of work organization based on the concept of physical construction flow, Bulletin of Civil Engineers, 1 (72), 87-93 (2019)

6. V. V. Sokolnikov, Modeling of organizational and technological reliability of construction, Bulletin of Civil Engineers, 4(69), 92-97 (2018)

7. V. V. Kostyuchenko, D. O. Kudinov, Organizational and technical modeling of design and construction systems, Engineering Bulletin of the Don, 3 (2012)

http://www.ivdon.ru/magazine/archive/n3y2012/988 (last accessed: 25.04.2021 г.)

8. A. R. Borodin, S. A. Barkalov, A. Sychev, Modeling of optimal priority of implementation of innovative projects, Bulletin of the Moscow Automobile and Road State Technical University (MADI), 4, 77-81 (2008) 
9. S. A. Bolotin, A. Kh. Dadar, K. V. Ivanov, D. T. Kurasova, Model of risk planning for untimely performance of work in POS on the basis of spatiotemporal analogy, Bulletin of Civil Engineers, 6(41), 69-75 (2013)

10. S. A. Bolotin, A. Kh. Dadar, I. S. Ptukhina, Improving the PERT method in statistical modeling of calendar plans, Bulletin of Civil Engineers, 2, 132-138 (2012)

11. S. A. Bolotin, A. Kh. Dadar, M. A. Kotovskaya, Model of spatial-temporal analogy in the optimization of the sequence of reconstructed objects, Civil Engineering Journal, 7, 51-57 (2013)

12. I. Yu. Meshchaninov, Improvement of the search model for optimal priority of object development, in proccedings of the 66th Scientific Conference of professors, teachers, researchers, engineers and postgraduates of SPbGASU, Saint Petersburg, 2009

13. V. V. Sobolev, Matematicheskoe modelirovanie i metody optimizatsii v proektirovanii organizatsii stroitelstva [Mathematical modeling and optimization methods in the design of construction organizations], The North Caucasus region. Series: Technical Sciences, 1(159), 106-109 (2011)

14. V. V.Sobolev, Mathematical modeling and optimization of the sequence of construction of objects according to the criterion of time, Izvestiya vysshikh uchebnykh zavedeniy. The North Caucasus region. Series: Technical Sciences, 3(161), 30-32 (2011)

15. V. Ya.Mishchenko, N. A. Ponyavina., A. N. Nazarov, Modeling of a stable organizational and technological system of reproduction of urban infrastructure, Scientific Bulletin of the Voronezh State University of Architecture and Civil Engineering. Series: High-tech. Ecology, 1, 74-80 (2010)

16. S. A. Bolotin, Simulation of calendar planning in building information modeling programs and regression detailing of construction duration norms, Engineering and construction magazine, 7(25), 82-86 (2011)

17. N. A.Goryaeva, A. O. Rybakova, Cloud technologies of interaction in design and construction, BST: Bulletin of construction equipment, 4(1004), 28-31 (2018)

18. S. A. Krivoy, A. I.Semin, A.V. Popov, B. O. Tebekin, The relationship of bim scenarios within the investment and development project, Construction of unique buildings and structures, 2(65), 20-39 (2018)

19. I. V.Karakozova, A. S. Pavlov, Creating a network model based on a universal sequence of construction works, Construction: science and education, 10(3), 1-16 (2020)

20. V. V. Sobolev, V. R. N. Gerasimenko, Z. K. Himishev, Information modeling of interrelations between the parameters of the work front, News of higher educational institutions. The North Caucasus region. Series: Technical Sciences, S1, 16-19 (2008)

21. V. V. Sobolev, Information modeling in the development of construction organization projects and work production projects, News of higher educational institutions. The North Caucasus region. Series: Technical Sciences, S1, 31-35 (2008)

22. I. G. Shepelev, Mathematical methods and models in construction («VSH», Moscow, 213, 1980)

23. B. Ya. Soviets, S. A.Yakovlev, Modeling of systems («VSH», Moscow, 270, 1985)

24. A. Webster, G. Sege, Differential equations in partial derivatives of mathematical physics, Ch. 1. (ONTI, Moscow, Leningrad, 285, 1934) 